\title{
MINERALIZAÇÃO DE NITROGÊNIO E ENXOFRE EM SOLOS BRASILEIROS SOB INFLUÊNCIA DA CALAGEM E FÓSFORO' ${ }^{1}$
}

\author{
CARLOS ALBERTO SILVA², FABIANO RIBEIRO DO VALE ${ }^{3}$, \\ SHARON JILL ANDERSON ${ }^{4}$ e ALEX RODRIGUES KOBAL ${ }^{5}$
}

\begin{abstract}
RESUMO - O presente estudo, desenvolvido em laboratório no período de outubro de 1995 a janeiro de 1996, objetivou avaliar a influência da calagem e fósforo (P) sobre a mineralização de nitrogênio (N) e enxofre (S) em sete solos, com ampla variabilidade nas características químicas e físicas. Os solos $(200 \mathrm{~g})$, numa etapa anterior à aplicação de $\mathrm{P}\left(\mathrm{KH}_{2} \mathrm{PO}_{4}\right.$ p.a. $)$, foram pré-incubados por sete dias com $\mathrm{CaCO}_{3}$ p.a. A seguir, foram incubados novamente, por mais 70 dias. Os teores de $\mathrm{N}$ $\left(\mathrm{NO}_{3}{ }^{-}+\mathrm{NH}_{4}^{+}\right)$e $\mathrm{S}\left(\mathrm{SO}_{4}{ }^{2-}\right)$ mineralizados em condições aeróbias foram avaliados a cada catorze dias, e notou-se neles uma dinâmica diferenciada, com o $\mathrm{N}$ mineral apresentando maior variação do que o sulfato. As quantidades de $\mathrm{N}$ mineralizado não se mostraram dependentes da acidez do solo, porém notou-se uma aceleração na mineralização do N com a calagem do Glei Húmico e do Latossolo Vermelho-Amarelo coletado no Rio de Janeiro. Ao contrário, a mineralização de S no Glei Pouco Húmico e no Latossolo Vermelho-Amarelo coletado no Rio de Janeiro foi influenciada pela correção da acidez do solo, que promoveu maior disponibilidade de sulfato nesses solos. Os teores de $\mathrm{N}$ e $\mathrm{S}$ mineralizados não foram influenciados pelos níveis de $\mathrm{P}$ disponível. A maior prevalência de nitrato e sulfato em alguns dos solos calcariados implica maior cuidado no estabelecimento da época de aplicação de corretivo.
\end{abstract}

Termos para indexação: nitrogênio mineral, amônio, nitrato, sulfato, nitrificação, organismos mineralizadores.

\section{NITROGEN AND SULFUR MINERALIZATION IN BRAZILIAN SOILS UNDER INFLUENCE OF LIMING AND PHOSPHORUS}

\begin{abstract}
This study was carried out under laboratory conditions, from October 1995 to January 1996, in order to evaluate the influence of liming and phosphorus $(\mathrm{P})$ application on nitrogen $(\mathrm{N})$ and sulfur (S) mineralization in seven Brazilian $(0-20 \mathrm{~cm})$ soils with a wide variability in chemical and physical properties. Soil samples, before fertilization with $\mathrm{P}\left(\mathrm{KH}_{2} \mathrm{PO}_{4}\right)$, were pre-incubated during seven days with $\mathrm{CaCO}_{3}$, and incubated again for another 70 days. The content of mineralized $\mathrm{N}$ $\left(\mathrm{NH}_{4}^{+}\right.$and $\left.\mathrm{NO}_{3}{ }^{-}\right)$and $\mathrm{S}\left(\mathrm{SO}_{4}^{2-}\right)$ were measured every other week, in aerobic conditions. Transformation of $\mathrm{N}$ and $\mathrm{S}$ showed different mineralization patterns from each other, with mineral $\mathrm{N}$ levels undergoing much greater variation than sulfate levels. Mineralized N amounts were not affected by soil acidity, although liming accelerated N mineralization in Humic Gley and Red-Yellow Latosol (colected in Rio de Janeiro) soils. Liming the soils to a $\mathrm{pH}$ around 6.0 resulted in increased net $\mathrm{S}$ mineralization in Low Humic Gley and Red-Yellow Latosol (colected in Rio de Janeiro) soils. Addition of superphosphate did not influence $\mathrm{N}$ and $\mathrm{S}$ mineralization processes. The greater prevalence of nitrate and sulphate in some of the limed soils suggests that the criterion used to choose time of lime application needs to be changed.
\end{abstract}

Index terms: mineral nitrogen, ammonium, nitrate, sulfate, nitrification, mineralizer organisms.

\footnotetext{
${ }^{1}$ Aceito para publicação em 7 de outubro de 1998.

${ }^{2}$ Eng. Agr., D.Sc., Embrapa-Centro Nacional de Pesquisa de Solos (CNPS), Rua Jardim Botânico, 1024, CEP 22460-000 Jardim Botânico, Rio de Janeiro, RJ. E-mail: csilva@cnps.embrapa.br

${ }^{3}$ Eng. Agr., Ph.D., Dep. de Ciência do Solo, Universidade Federal de Lavras (UFLA), Caixa Postal 37, CEP 37200-000 Lavras, MG.

${ }^{4}$ Geólogo, Ph.D., California State University at Monterey Bay. 93955, Seaside, USA. E-mail: sharon-anderson@monterey.edu

${ }^{5}$ Estudante do Curso de Agronomia, UFLA.
}

\section{INTRODUÇÃO}

Os solos brasileiros são, na sua maioria, caracterizados pelo avançado grau de intemperização, pela elevada acidez e baixa disponibilidade de nutrientes (Goedert, 1983). Nessas condições, a produtividade potencial das culturas pode ser reduzida, a menos que se proceda a correção da acidez do solo. A aplicação de P se constitui também uma etapa essencial na obtenção 
de altas produtividades, pelo fato de a maioria das culturas responder favoravelmente à aplicação desse nutriente e ser elevada a capacidade dos solos brasileiros em fixá-lo (Lopes, 1983).

Os efeitos da aplicação de calcário e de $\mathrm{P}$ sobre algumas características químicas dos solos e sobre o crescimento das plantas são bastante conhecidos, contudo poucos têm sido os estudos voltados à investigação da influência dessas práticas sobre a mineralização de $\mathrm{N}$ e $\mathrm{S}$ nativos. $\mathrm{O}$ processo da mineralização consiste na conversão de formas orgânicas de $\mathrm{N}$ e $\mathrm{S}$ em formas inorgânicas, amônio e sulfato, respectivamente. A intensidade desse processo se mostra bastante influenciada por fatores bióticos e abióticos (Black, 1968). Entre esses, há um destaque em relação ao $\mathrm{pH}$ do solo, pelo fato de a correção da acidez estar associada a um aumento na disponibilidade de $\mathrm{N}$ para as plantas, em função das maiores quantidades de $\mathrm{N}$ mineralizadas nos solos calcariados (Haynes, 1986; Silva et al., 1994). Há que se considerar que a intensidade desse efeito varia também com o tipo de solo, podendo ser apenas temporária em alguns agroecossistemas (Nyborg \& Hoyt, 1978; Silva et al., 1994).

$\mathrm{O}$ aumento de $\mathrm{pH}$ e a maior disponibilidade de cátions trocáveis nos solos calcariados têm-se mostrado também eficientes em aumentar as quantidades de $\mathrm{S}$ orgânico convertido em sulfato (Wainwright, 1984; Alvarez, 1988). Nos solos menos sujeitos à lixiviação, maiores teores de $\mathrm{S}$ mineralizado podem representar maior disponibilidade desse nutriente às plantas. Nos solos mais arenosos e permeáveis, onde o sulfato se mostra majoritariamente presente na solução, a aplicação de calcário pode resultar em maior fluxo desse nutriente para as camadas mais profundas, inacessíveis às raízes das plantas, e em um maior agravamento dos sintomas de deficiência de $\mathrm{S}$, cada vez mais freqüentes nos solos brasileiros (Alvarez, 1988).

Quanto à influência do $\mathrm{P}$ sobre a mineralização do N, poucos têm sido os estudos efetuados, e, na maioria das vezes, com resultados não conclusivos. A análise desses estudos revelou que a maior disponibilidade de $\mathrm{P}$ para solos australianos sob floresta resultou numa elevação nas taxas de $\mathrm{N}$ mineralizado e em maiores teores de $\mathrm{N}$ absorvido pelas plantas, por períodos que se estenderam a até dois anos (Falkiner et al., 1993). Resultados semelhantes foram observados por Munevar \& Wollum (1977) em solos vulcânicos, muito embora, neste caso, o processo de mineralização de $\mathrm{N}$ só tenha se mostrado controlado pelos níveis de P quando da aplicação de doses desse nutriente superiores a $1.200 \mathrm{mg} / \mathrm{kg}$ de solo. De certa forma, os resultados alcançados pelos últimos autores concordam com aqueles apresentados por Coelho (1992), que observou não ser o processo de mineralização de $\mathrm{N}$ influenciado pela aplicação de solução nutritiva contendo $2,5 \mathrm{~mol} / \mathrm{L} \mathrm{de} \mathrm{Ca}\left(\mathrm{H}_{2} \mathrm{PO}_{4}\right)$. Nos estudos relatados, menores taxas de mineralização de N têm sido, invariavelmente, associadas à baixa atividade dos organismos mineralizadores nos solos com deficiência de P (Haynes, 1986).

Em alguns estudos (McGill \& Cole, 1981; Sakadevan et al., 1993), as quantidades de N e $\mathrm{S}$ mineralizadas não se mostram associadas aos teores totais desses elementos no solo. Maiores teores de $\mathrm{N}$ mineralizado não implicam necessariamente maior conversão de $\mathrm{S}$ orgânico a sulfato, pelo fato de os processos de mineralização de $\mathrm{N}$ e $\mathrm{S}$ se mostrarem dissociados e com taxas diferenciadas (Tabatabai \& Al-Khafaji, 1980). Além disso, parte das frações orgânicas contendo $\mathrm{S}$ pode ser mineralizada sem a mediação da biomassa microbiana, o que sugere ser a intensidade de mineralização de $\mathrm{S}$ menos dependente da disponibilidade de $\mathrm{C}$ no solo, em relação à de $\mathrm{N}$, já que pode haver oxidação de $\mathrm{S}$ por processos bioquímicos, via hidrólise enzimática de frações sulfuradas do tipo éster (Sakadevan et al., 1993).

Diante de todas essas considerações, percebe-se que estudos direcionados a um melhor entendimento das transformações de $\mathrm{N}$ e $\mathrm{S}$ no solo se mostram muito úteis na modelagem do ciclo desses elementos em diferentes sistemas agrícolas, tendo em vista uma maximização no uso desses nutrientes pelas plantas e, por pressuposto, a busca de uma maior eficiência no uso de adubos nitrogenados e sulfurados.

O presente estudo teve por objetivo avaliar os efeitos da calagem e da adição de $\mathrm{P}$ sobre a 
mineralização de $\mathrm{N}$ e $\mathrm{S}$, em solos com ampla variabilidade nas características químicas e físicas.

\section{MATERIAL E MÉTODOS}

Foram utilizadas amostras de sete solos $(0-20 \mathrm{~cm})$, as quais, após a coleta, foram secadas ao ar e tamisadas em peneira de $2 \mathrm{~mm}$. A caracterização química e a textura dos solos Glei Húmico (HGH), Glei Pouco Húmico (HGP), Latossolo Roxo (LR), Latossolo Vermelho-Amarelo (LV1), Latossolo Vermelho-Amarelo (LV2), Podzólico Vermelho-Amarelo (PV) e Cambissolo (C) encontram-se na Tabela 1. Os solos HGH e LR foram coletados em Lavras, MG, o HGP, LV2 e PV no município do Rio de Janeiro, RJ, o LV1, em Itumirim, MG, e o C, no município de Nazareno, MG. O desenvolvimento do presente estudo se deu no Departamento de Ciência do Solo da UFLA, Lavras, MG, no período de outubro de 1995 a janeiro de 1996.

As análises químicas foram efetuadas segundo os métodos descritos pela Embrapa (1979), quanto ao pH em água e em $\mathrm{CaCl}_{2}, \mathrm{Ca}^{2+}, \mathrm{Mg}^{2+}$ e $\mathrm{K}^{+}$, sendo o $\mathrm{P}$ extraído pelo método da resina (Raij \& Quaggio, 1983). Os teores de $\mathrm{C}$ foram determinados segundo método descrito por Raij \& Quaggio (1983), quantificando-se este elemento pelo método da colorimetria. No HGH, o teor de $\mathrm{C}$ total foi determinado pelo método de Walkley-Black (Raij \& Quaggio, 1983). A acidez potencial (H+Al) foi determinada indiretamente pelo método do $\mathrm{pH}$ SMP, sendo a capacidade de troca catiônica (CTC) em pH 7,0 estimada mediante a expressão: $\mathrm{CTC}=(\mathrm{H}+\mathrm{Al})+$ $\mathrm{Ca}^{2+}+\mathrm{Mg}^{2+}+\mathrm{K}^{+}$, em cmol( $(+) / \mathrm{dm}^{3}$. O N total foi analisado conforme o método descrito por Bremner \& Mulvaney
(1982), e a análise granulométrica feita de acordo com Camargo et al. (1986). O S total foi determinado através da combustão $\left(500^{\circ} \mathrm{C}\right)$ de uma mistura de $2,5 \mathrm{~g}$ de solo e $1,0 \mathrm{~g}$ de $\mathrm{NaHCO}_{3}$ p.a. a $500^{\circ} \mathrm{C}$, conforme Bardsley \& Lancaster (1960). O S ( $\left.\mathrm{SO}_{4}{ }^{2-}\right)$ foi extraído com solução de $\mathrm{Ca}\left(\mathrm{H}_{2} \mathrm{PO}_{4}\right)_{2} \cdot \mathrm{H}_{2} \mathrm{O}$ em ácido acético $2 \mathrm{~N}$ contendo $500 \mathrm{ppm}$ de $\mathrm{P}$ e determinado por turbidimetria, seguindo-se Nascimento \& Morelli (1980).

A necessidade de calagem foi determinada baseando-se em curvas de neutralização (Tabela 2) dos diferentes solos, visando-se atingir valor de $\mathrm{pH}$ em água igual a 6,0, à exceção do $\mathrm{HGH}$, cujo valor de $\mathrm{pH}$ visado foi 5,2. As declividades das curvas de neutralização demonstraram maior e menor poder-tampão, respectivamente, do LR e do LV1. Coincidentemente, o LR foi o solo com maior teor de argila, sendo o LV1 o solo mais arenoso e com o menor conteúdo de C (Tabela 1). Os solos, após a calagem (Tabela 2), foram pré-incubados por sete dias, numa etapa anterior à sua fertilização com $\mathrm{P}\left(\mathrm{KH}_{2} \mathrm{PO}_{4}\right.$ p.a.). A adição de $\mathrm{P}$ foi efetuada visando-se atingir níveis de $\mathrm{P}$ disponível - extraído pela resina próximos de $25 \mathrm{mg} / \mathrm{dm}^{3}$ de solo, baseando-se em curvas de calibração previamente obtidas.

$\mathrm{O}$ experimento, considerando-se cada tipo de solo em separado, entre os sete estudados, constou de quatro tratamentos: sem calagem e sem $P$ (Test), com calagem e sem $\mathrm{P}(\mathrm{Cal})$, com $\mathrm{P}$ e sem calagem (P) e com calagem e $\mathrm{P}(\mathrm{Cal}+\mathrm{P})$, em delineamento experimental inteiramente casualizado, com três repetições.

$\mathrm{Na}$ montagem do experimento, transferiram-se para copos de plástico de $250 \mathrm{~mL} / 200 \mathrm{~g}$ de solo pré-incubado com e sem calcário, sendo essas amostras, numa etapa posterior à aplicação de $\mathrm{P}$ (quando pertinente), incubadas novamente por períodos contínuos de $14,28,42,56$ e

TABELA 1. Caracterização química e textura dos sete materiais de solo utilizados no estudo, antes da aplicação dos tratamentos.

\begin{tabular}{lccrcccccccc}
\hline Solo $^{1}$ & $\mathrm{MO}^{2}$ & $\mathrm{pH}_{\mathrm{CaCl}_{2}}$ & $\mathrm{CTC}$ & $\mathrm{H}+\mathrm{Al}$ & $\mathrm{Ca}^{2+}$ & $\mathrm{Mg}^{2+}$ & $\mathrm{P}$ & $\mathrm{K}^{+}$ & Argila & Silte & Areia \\
\hline & $\left(\mathrm{g} / \mathrm{dm}^{3}\right)$ & & $-------------\left(\mathrm{mol}_{\mathrm{c}} / \mathrm{dm}^{3}\right)-------------$ & $--\left(\mathrm{mg} / \mathrm{dm}^{3}\right)--$ & $-----------(\mathrm{g} / \mathrm{kg})----------$ \\
HGH & 236,0 & 4,1 & 12,5 & 11,0 & 1,0 & 0,5 & 13 & 17 & 190 & 670 & 140 \\
HGP & 34,0 & 4,0 & 10,7 & 7,0 & 2,4 & 1,2 & 11 & 32 & 350 & 390 & 260 \\
LR & 49,0 & 4,0 & 10,1 & 8,8 & 0,7 & 0,5 & 14 & 32 & 550 & 110 & 340 \\
LV1 & 14,0 & 4,2 & 4,1 & 2,3 & 1,0 & 0,7 & 9 & 32 & 190 & 30 & 780 \\
LV2 & 45,0 & 3,5 & 16,4 & 15,3 & 0,6 & 0,4 & 13 & 40 & 190 & 100 & 710 \\
PV & 22,1 & 4,5 & 9,4 & 4,5 & 2,8 & 2,0 & 11 & 37 & 370 & 190 & 440 \\
C & 20,0 & 4,1 & 4,5 & 3,2 & 0,8 & 0,4 & 9 & 29 & 210 & 150 & 640 \\
\hline
\end{tabular}

${ }^{1}$ HGH: Glei Húmico; HGP: Glei Pouco Húmico; LR: Latossolo Roxo; LV1: Latossolo Vermelho-Amarelo (Itumirim); LV2: Latossolo Vermelho-Amarelo (Rio de Janeiro); PV: Podzólico Vermelho-Amarelo; C: Cambissolo.

2 Teor de matéria orgânica no solo. 
70 dias. Todos os copos foram cobertos com folhas de alumínio, com a finalidade de evitar perdas excessivas de água por evaporação. A incubação foi realizada em laboratório à temperatura ambiente $\left(21\right.$ a $\left.29^{\circ} \mathrm{C}\right)$, sendo o grau de umidade dos solos mantido próximo de $60 \%$ do volume total de poros.

No início e ao término de cada período de incubação, determinaram-se os teores de $\mathrm{N}$ mineral em cada tratamento, através da extração do nitrato e do amônio com $\mathrm{KCl} 1 \mathrm{M}$ e destilação dessas formas de $\mathrm{N}$ por arraste de vapores (Keeney \& Nelson, 1982). O cálculo do N mineralizado foi efetuado deduzindo-se do $\mathrm{N}$ mineral determinado ao término de cada período de incubação o amônio e nitrato quantificado no início de cada período. Adotou-se procedimento semelhante na avaliação do S mineralizado, quantificando-se os teores de sulfato (Nascimento \& Morelli, 1980) no início e ao término de cada período de incubação.

\section{RESULTADOS E DISCUSSÃO}

Por ocasião da instalação e término do presente estudo, os solos apresentavam ampla variabilidade nas propriedades químicas, as quais são apresentadas na Tabela 3. Em termos gerais, foi notada uma diminuição nos valores de $\mathrm{pH}$ ao término dos 70 dias de incubação, muito provavelmente relacionada aos processos de mineralização e nitrificação, processos sabidamente acidificantes. A análise dos dados apresentados na Tabela 3 revelou também tendência de aumento da $\mathrm{CTC}$ a pH 7,0 no tratamento $\mathrm{P}$. Acréscimos na capacidade dos solos fosfatados em reter cátions são associados ao decréscimo, pelos íons fosfatos, no número de cargas positivas, e maior densidade de cargas negativas nas superfícies de sorção (Guadalix \& Pardo, 1994). Nos solos com os maiores teores de $\mathrm{C}$, foi verificada uma diminuição nos níveis de $\mathrm{Al}$ trocável. É provável que, no curso dos processos de mineralização e decomposição da matéria orgânica, estejam sendo liberadas frações orgânicas com maior capacidade de neutralizar o Al tóxico. A adsorção de Al na superfície do material orgânico, a precipitação do $\mathrm{Al}$ pelo aumento de $\mathrm{pH}$ devido às reações de troca entre os ânions orgânicos liberados durante a decomposição da matéria orgânica e hidróxidos terminais de óxidos de ferro e alumínio e a complexação de $\mathrm{Al}$ por ácidos orgânicos de baixo peso molecular e íons fulvatos são os principais mecanismos envolvidos na reação do material orgânico na acidez do solo (Miyazawa et al.,1993; Smith et al., 1995).

Os teores de $\mathrm{C}$ variaram entre os diferentes tipos de solo, sendo o valor mais alto observado no HGH, e o mais baixo, no solo mais arenoso, o LV1 (Tabela 4). Os teores de $\mathrm{N}$ total apresentaram distribuição semelhante à de $\mathrm{C}$, mostrando-se positivamente correlacionados com os teores deste elemento nos sete materiais de solo $\left(\mathrm{r}=0,99^{* *}\right)$. Os teores totais de $\mathrm{C}$ se mostraram também muito associados aos de $\mathrm{S}$ total $\left(\mathrm{r}=0,84^{* *}\right)$, quando se excluíram os teores totais de $\mathrm{C}$ e $\mathrm{S}$ presentes no $\mathrm{HGH}$.

TABELA 2. Curvas de neutralização dos solos estudados.

\begin{tabular}{llcrr}
\hline Solo $^{1}$ & \multicolumn{1}{c}{ Equação $^{2}$} & $\mathrm{r}^{2}$ & $\mathrm{~b}^{3}$ & $\mathrm{CaCO}_{3}{ }^{4}$ \\
\hline HGH & $\mathrm{pH}=4,8691+0,005884\left(\mathrm{CaCO}_{3}\right)$ & 0,95 & 0,005884 & 56,24 \\
HGP & $\mathrm{pH}=4,779+0,006338\left(\mathrm{CaCO}_{3}\right)$ & 0,99 & 0,006338 & 192,65 \\
LR & $\mathrm{pH}=4,806+0,004629\left(\mathrm{CaCO}_{3}\right)$ & 0,97 & 0,004629 & 257,94 \\
LV1 & $\mathrm{pH}=5,1685+0,01784\left(\mathrm{CaCO}_{3}\right)$ & 0,99 & 0,017840 & 46,61 \\
LV2 & $\mathrm{pH}=4,2991+0,007691\left(\mathrm{CaCO}_{3}\right)$ & 0,95 & 0,007691 & 221,15 \\
PV & $\mathrm{pH}=5,099+0,01521\left(\mathrm{CaCO}_{3}\right)$ & 0,96 & 0,015210 & 59,24 \\
C & $\mathrm{pH}=4,975+0,01418\left(\mathrm{CaCO}_{3}\right)$ & 0,98 & 0,014180 & 72,28 \\
\hline
\end{tabular}

${ }^{1}$ HGH: Glei Húmico; HGP: Glei Pouco Húmico; LR: Latossolo Roxo; LV1: Latossolo Vermelho-Amarelo (Itumirim); LV2: Latossolo Vermelho-Amarelo (Rio de Janeiro); PV: Podzólico Vermelho-Amarelo; C: Cambissolo.

${ }^{2}$ Relaciona quantidade de $\mathrm{CaCO}_{3}$ p.a. (mg/100 g de solo) necessária para atingir um dado valor de $\mathrm{pH}$ do solo.

${ }^{3}$ Declividade das curvas de neutralização.

${ }^{4}$ Quantidade de $\mathrm{CaCO}_{3}$ p.a. (mg/100 g de solo) necessária para se atingir pH=5,2 no HGH e 6,0 nos solos HGP, LR, LV1, LV2, PV e C. 
TABELA 3. Análise química de amostras da camada superficial $(0-20 \mathrm{~cm})$ dos sete materiais de solo, após a aplicação dos tratamentos, no início (i) e após 70 dias de incubação (f).

\begin{tabular}{|c|c|c|c|c|c|c|c|c|c|c|c|}
\hline Solo ${ }^{1}$ & Trat $^{2}$ & $\mathrm{pH}_{\mathrm{H}_{2} \mathrm{Oi}}$ & $\mathrm{pH}_{\mathrm{H}_{2} \mathrm{Of}}$ & $\mathrm{CTC}_{\mathrm{i}}$ & $\mathrm{CTC}_{\mathrm{f}}$ & $\mathrm{P}_{\mathrm{i}}$ & $P_{f}$ & $\mathrm{Al}^{3+}{ }_{\mathrm{i}}$ & $\mathrm{Al}_{\mathrm{f}}^{3+}$ & $\mathrm{V}_{\mathrm{i}}$ & $\mathrm{V}_{\mathrm{f}}$ \\
\hline & & & & \multicolumn{2}{|c|}{$---\left(\mathrm{cmol}_{\mathrm{c}} / \mathrm{dm}^{3}\right)---$} & \multicolumn{2}{|c|}{$---\left(\mathrm{mg} / \mathrm{dm}^{3}\right)---$} & \multicolumn{2}{|c|}{$---\left(\mathrm{cmol}_{\mathrm{c}} / \mathrm{dm}^{3}\right)---$} & \multicolumn{2}{|c|}{-----(\%)----. } \\
\hline \multirow[t]{4}{*}{$\mathrm{HGH}$} & Test & 4,8 & 4,6 & 18,5 & 17,6 & 11 & 15 & 3,0 & 2,2 & 10 & 8 \\
\hline & $\mathrm{Cal}$ & 5,2 & 5,1 & 15,4 & 16,0 & 13 & 17 & 1,5 & 0,9 & 28 & 23 \\
\hline & $\mathrm{P}$ & 4,8 & 4,6 & 16,7 & 19,3 & 36 & 32 & 2,7 & 2,0 & 10 & 6 \\
\hline & $\mathrm{Cal}+\mathrm{P}$ & 5,2 & 5,1 & 15,1 & 17,4 & 35 & 38 & 1,4 & 1,1 & 25 & 22 \\
\hline \multirow[t]{4}{*}{ HGP } & Test & 4,6 & 4,5 & 9,9 & 14,8 & 10 & 10 & 1,9 & 1,1 & 36 & 26 \\
\hline & Cal & 6,0 & 5,6 & 9,8 & 11,9 & 10 & 11 & 0,1 & 0,2 & 76 & 63 \\
\hline & $\mathrm{P}$ & 4,7 & 4,5 & 11,0 & 13,8 & 17 & 18 & 1,8 & 1,2 & 43 & 29 \\
\hline & $\mathrm{Cal}+\mathrm{P}$ & 6,0 & 5,6 & 9,8 & 11,9 & 18 & 16 & 0,1 & 0,1 & 76 & 66 \\
\hline \multirow[t]{4}{*}{ LR } & Test & 4,6 & 4,5 & 10,4 & 12,8 & 9 & 8 & 2,4 & 1,9 & 7 & 4 \\
\hline & $\mathrm{Cal}$ & 5,7 & 5,5 & 9,5 & 11,2 & 9 & 9 & 0,1 & 0,1 & 54 & 44 \\
\hline & $\mathrm{P}$ & 4,6 & 4,5 & 11,4 & 13,1 & 40 & 33 & 2,1 & 1,7 & 14 & 6 \\
\hline & $\mathrm{Cal}+\mathrm{P}$ & 5,6 & 5,5 & 9,7 & 11,0 & 42 & 31 & 0,1 & 0,1 & 54 & 47 \\
\hline \multirow[t]{4}{*}{$\overline{\text { LV1 }}$} & Test & 5,0 & 4,9 & 2,7 & 3,6 & 10 & 11 & 0,6 & 0,4 & 22 & 19 \\
\hline & $\mathrm{Cal}$ & 5,8 & 5,8 & 3,1 & 3,9 & 10 & 12 & 0,1 & 0,1 & 54 & 46 \\
\hline & $\mathrm{P}$ & 5,0 & 5,0 & 2,8 & 3,5 & 22 & 21 & 0,5 & 0,4 & 22 & 26 \\
\hline & $\mathrm{Cal}+\mathrm{P}$ & 5,8 & 5,7 & 2,9 & 3,9 & 24 & 25 & 0,0 & 0,1 & 55 & 46 \\
\hline \multirow[t]{4}{*}{ LV2 } & Test & 4,4 & 4,4 & 10,4 & 11,9 & 4 & 7 & 3,2 & 2,3 & 15 & 8 \\
\hline & Cal & 5,7 & 5,6 & 8,2 & 8,2 & 5 & 8 & 0,1 & 0,1 & 61 & 51 \\
\hline & $\mathrm{P}$ & 4,4 & 4,4 & 10,4 & 11,8 & 20 & 17 & 2,6 & 2,2 & 15 & 7 \\
\hline & $\mathrm{Cal}+\mathrm{P}$ & 5,6 & 5,6 & 8,7 & 9,0 & 14 & 21 & 0,0 & 0,1 & 62 & 51 \\
\hline \multirow[t]{4}{*}{ PV } & Test & 4,9 & 5,0 & 5,8 & 6,0 & 4 & 7 & 0,4 & 0,3 & 43 & 42 \\
\hline & Cal & 5,9 & 5,4 & 5,7 & 6,3 & 4 & 7 & 0,0 & 0,1 & 59 & 59 \\
\hline & $\mathrm{P}$ & 4,9 & 4,9 & 5,8 & 5,9 & 14 & 12 & 0,5 & 0,3 & 40 & 40 \\
\hline & $\mathrm{Cal}+\mathrm{P}$ & 5,9 & 5,5 & 5,7 & 6,3 & 18 & 13 & 0,0 & 0,1 & 60 & 60 \\
\hline \multirow[t]{4}{*}{$\mathrm{C}$} & Test & 4,8 & 4,8 & 3,9 & 4,5 & 4 & 7 & 1,0 & 0,9 & 18 & 16 \\
\hline & $\mathrm{Cal}$ & 5,8 & 5,7 & 4,0 & 4,3 & 4 & 6 & 0,0 & 0,1 & 57 & 47 \\
\hline & $\mathrm{P}$ & 4,7 & 4,7 & 4,1 & 4,3 & 20 & 15 & 0,9 & 1,0 & 17 & 17 \\
\hline & $\mathrm{Cal}+\mathrm{P}$ & 5,8 & 5,8 & 4,2 & 4,3 & 20 & 14 & 0,0 & 0,1 & 52 & 47 \\
\hline
\end{tabular}

${ }^{1}$ HGH: Glei Húmico; HGP: Glei Pouco Húmico; LR: Latossolo Roxo; LV1: Latossolo Vermelho-Amarelo (Itumirim); LV2: Latossolo Vermelho-Amarelo (Rio de Janeiro); PV: Podzólico Vermelho-Amarelo; C: Cambissolo.

2 Test: sem calagem e sem fósforo; Cal: com calagem e sem fósforo; P: com fósforo e sem calagem; Cal+P: com calagem e fósforo.

O $\mathrm{S}$ total apresentou valor médio de $157 \mathrm{mg} / \mathrm{dm}^{3}$ de solo, sendo o valor mais baixo encontrado no LV1 e o mais alto no LV2. Surpreendentemente, as maiores reservas de $\mathrm{C}$ e $\mathrm{N}$ observadas para o HGH não estiveram associadas ao maior armazenamento de $\mathrm{S}$ nesse solo. Os teores de $\mathrm{S}$ total se situaram muito próximos aos teores de S orgânico avaliados em solos do Rio Grande do Sul, porém se mostraram inferiores aos teores totais de $\mathrm{S}$ determinados nesses mesmos solos (Nascimento \& Morelli, 1980). De certa forma, os dados apresentados pelos últimos autores se aproximam daqueles observados para solos de região temperada (Tabatabai \& Al-Khafaji, 1980) e denotam maior contribuição da fase mineral para os teores totais de $\mathrm{S}$ nos solos oriundos da Região Sul do Brasil. É provável que os estoques de $\mathrm{S}$ total dos sete materiais de solos estudados estejam mais associados à fase orgânica, pelo fato de se tratar de solos mais intemperizados, com predominância de argila do tipo 1:1. Os dados de $\mathrm{S}$ disponível se mostraram muito próximos aos apresentados por 
TABELA 4. Teores totais de carbono, formas de nitrogênio e enxofre e relações entre esses elementos nos sete solos utilizados no estudo.

\begin{tabular}{|c|c|c|c|c|c|c|c|}
\hline Solo $^{1}$ & $\mathrm{C}^{2}$ & $\mathrm{~N}^{3}$ & $S^{4}$ & $\mathrm{~N}_{\text {mineral }}{ }^{5}$ & $\mathrm{~S}\left(\mathrm{SO}_{4}{ }^{2-}\right)$ & $\mathrm{C}: \mathrm{N}$ & $\mathrm{C}: \mathrm{N}: \mathrm{S}$ \\
\hline & \multicolumn{5}{|c|}{ 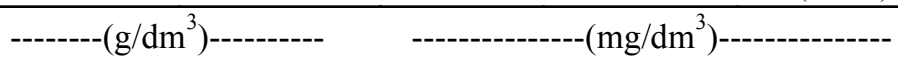 } & & \\
\hline $\mathrm{HGH}$ & 136,9 & 9,53 & 116 & 91,8 & 4,9 & 14,4 & $1180: 82: 1$ \\
\hline HGP & 19,7 & 2,10 & 171 & 18,1 & 31,6 & 9,4 & $115: 12: 1$ \\
\hline LR & 28,4 & 3,10 & 200 & 17,2 & 3,0 & 9,2 & $142: 16: 1$ \\
\hline LV1 & 8,1 & 0,90 & 69 & 10,3 & 4,9 & 9,0 & $117: 13: 1$ \\
\hline LV2 & 26,1 & 3,30 & 273 & 65,2 & 8,0 & 7,9 & $96: 12: 1$ \\
\hline PV & 12,8 & 1,58 & 179 & 13,9 & 7,0 & 8,1 & 72:09:1 \\
\hline $\mathrm{C}$ & 11,6 & 1,40 & 88 & 6,6 & 8,8 & 8,3 & $132: 16: 1$ \\
\hline
\end{tabular}

${ }^{1}$ HGH: Glei Húmico; HGP: Glei Pouco Húmico; LR: Latossolo Roxo; LV1: Latossolo Vermelho-Amarelo (Itumirim); LV2: Latossolo Vermelho-Amarelo (Rio de Janeiro); PV: Podzólico Vermelho-Amarelo; C: Cambissolo.

2 Teor total de carbono no solo.

3 Teor total de nitrogênio no solo.

4 Teor total de enxofre orgânico no solo.

5 Teores de nitrogênio $\left(\mathrm{NH}_{4}^{+}+\mathrm{NO}_{3}^{-}\right)$mineral presentes nos materiais de solo no início da incubação.

Nascimento \& Morelli (1980), à exceção do HGP, que apresentou altos teores de sulfato. De modo geral, os teores de sulfato estiveram abaixo do valor crítico recomendado para a maioria das culturas, $10 \mathrm{mg} / \mathrm{kg}$ de solo, e evidenciam a alta possibilidade de reposta à aplicação de $\mathrm{S}$ na maioria dos solos estudados, principalmente no LR, LV1 e HGH.

A relação C:N englobou valores próximos de 10:1, à exceção do solo de várzea $\mathrm{HGH}$, que apresentou relação $\mathrm{C}: \mathrm{N}$ mais alta, muito possivelmente devido à presença de frações orgânicas menos humificadas, preservadas pelo excesso de água no HGH (Tabela 4). As diferenças nas relações $\mathrm{C}: \mathrm{N}$ verificadas nos sete solos, de certa forma, evidenciam o grau de humificação diferenciado da matéria orgânica. Revelam também que, à medida que a intensidade de decomposição da matéria orgânica avança no sentido de equilíbrio das frações orgânicas com o solo, há uma perda de $\mathrm{C}$ do sistema solo-planta e uma concentração relativa de $\mathrm{N}$. Os valores de relação C:N:S estão de acordo com os valores apresentados por Nascimento \& Morelli (1980) e por Schnitzer \& Khan (1972). O único solo a diferir dos demais foi o $\mathrm{HGH}$, que apresentou uma relação C:N:S muito alta.

Na Fig. 1 são apresentados os teores de $\mathrm{N}$ mineralizado, na ausência ou presença de calcário e $\mathrm{P}$, considerando-se a incubação dos sete solos por intervalos seguidos de 14, 28, 42, 56 e 70 dias. Os dados apresentados se referem às quantidades líquidas de $\mathrm{N}$ mineralizado, refletindo o balanço entre os processos de mineralização e imobilização. Valores negativos demonstram serem as quantidades de $\mathrm{N}$ imobilizado maiores do que aquelas de $\mathrm{N}$ mineralizado. Verificou-se que a taxa de mineralização do $\mathrm{N}$ decresceu com o tempo de incubação, sendo, no geral, maior nos períodos iniciais de incubação (Fig. 1), o que está de acordo com Silva et al. (1994) e Stanford \& Smith (1972). No LV1, C e LV2, as taxas de mineralização foram muito baixas nos períodos iniciais de incubação, sendo observado, após 42 dias de incubação, um aumento nas quantidades de $\mathrm{N}$ mineralizado. Os padrões de liberação de $\mathrm{N}$ mineralizado verificados no LR e HGP foram muito semelhantes, sendo observado nesses dois solos taxas de mineralização de $\mathrm{N}$ similares nos cinco períodos de incubação (Fig. 1).

No $\mathrm{HGH}$, o processo de mineralização do $\mathrm{N}$ se mostrou dependente dos tratamentos, sendo notado, nos primeiro 28 dias de incubação, um aumento nas quantidades líquidas de $\mathrm{N}$ mineralizado nas amostras com calcário, e nos cinco períodos de incubação foi verificado mineralização líquida de N. Contudo, nos períodos de incubação de $42 \mathrm{e}$ 56 dias, foram observados níveis maiores de $\mathrm{N}$ imobilizado, com concomitante diminuição nas quantidades líquidas de $\mathrm{N}$ mineralizado. Novamente, após 52 dias e ao término de 70 dias de incubação, foi 
verificado diminuição nos níveis de $\mathrm{N}$ imobilizado, sendo notado também maior quantidade de $\mathrm{N}$ mineralizado no HGH, na presença de calcário. A prática da calagem no LV2 concorreu também para um aumento significativo nas quantidades de $\mathrm{N}$ mineralizado nos períodos de 42 e 56 dias de incubação.
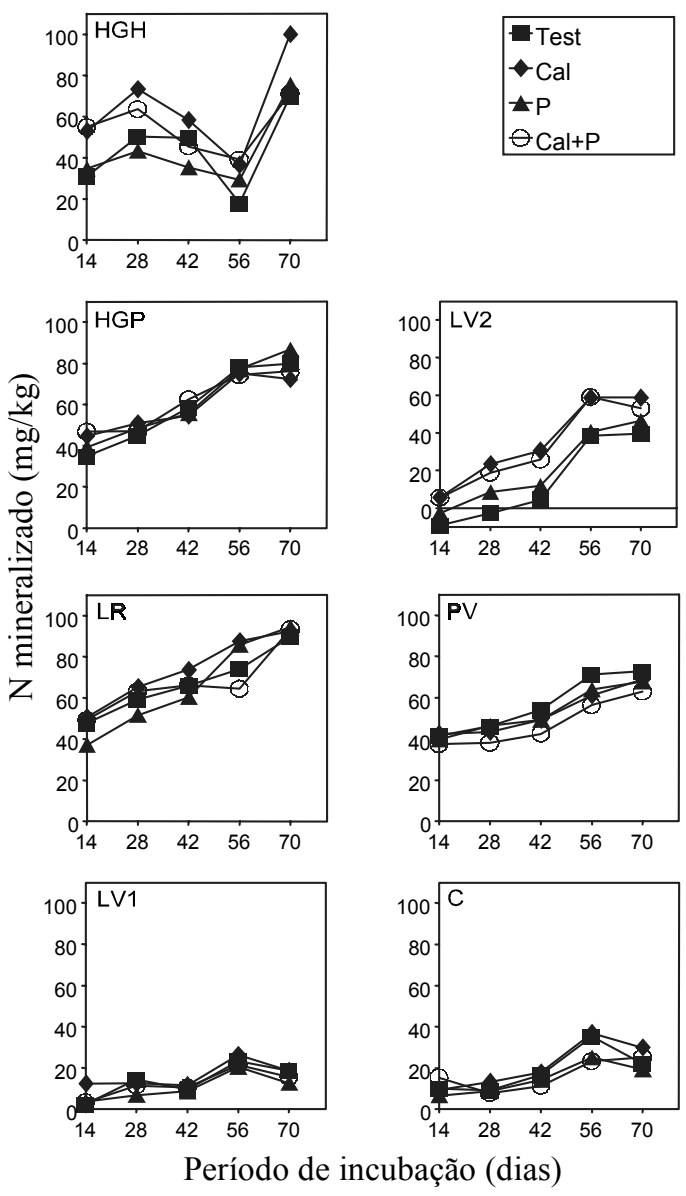

FIG. 1. Mineralização líquida de nitrogênio, ao término dos cinco períodos de incubação, sob influência da calagem e da adição de fósforo. HGH: Glei Húmico; HGP: Glei Pouco Húmico; LR: Latossolo Roxo; LV1: Latossolo Vermelho-Amarelo (Itumirim); LV2: Latossolo Vermelho-Amarelo (Riode Janeiro); PV: Podzólico VermeIho-Amarelo; C: Cambissolo.
Em termos gerais, a correção da acidez do solo não resultou em aumento nas quantidades de $\mathrm{N}$ mineralizado nos sete solos estudados, concorrendo, sim, para maior aceleração no processo de mineralização, provavelmente devido a uma diminuição nas quantidades de $\mathrm{N}$ imobilizado. De modo geral, os dados obtidos estão de acordo com os apresentados por Dancer et al. (1973) e Nyborg \& Hoyt (1978), os quais observaram efeito temporário da calagem no sentido de acelerar o processo de mineralização de N. Discordam, porém, dos resultados apresentados por Silva et al. (1994), os quais verificaram maiores teores de $\mathrm{N}$ mineralizado nos materiais de solo calcariados. $\mathrm{O}$ efeito da calagem em aumentar as quantidades de $\mathrm{N}$ mineralizado tem sido associado a uma elevação nos valores de $\mathrm{pH}$ e nos teores de cátions trocáveis, e à diminuição do Al tóxico, fatores esses que aumentam a atividade de organismos mineralizadores (Dancer et al., 1973; Nyborg \& Hoyt, 1978; Silva et al., 1994). Maiores taxas de $\mathrm{N}$ mineralizado em solos com calagem podem estar associadas ainda à maior degradação de compostos fenólicos inibitórios à decomposição da matéria orgânica (Marschner \& Wilczynski, 1991). Uma maior presença de frações orgânicas ligadas ao Ca nos solos corrigidos, em comparação com essas mesmas moléculas ligadas ao $\mathrm{Al}$, pode também resultar em maior decomposição da matéria orgânica e, por conseguinte, em maior mineralização do N (Mendonça \& Rowell, 1994).

As quantidades de $\mathrm{N}$ mineralizado no LR, HGP e PV não foram influenciadas pela correção da acidez do solo, tampouco pela adição de P. Resultados semelhantes foram verificados para os solos LV1 e C, sendo notado também nesses solos uma intensidade muito reduzida da mineralização de $\mathrm{N}$, o que revela a baixa capacidade do LV1 e C em suprir $\mathrm{N}$ às plantas.

A quantificação dos teores de nitrato, ao término de 70 dias de incubação, revelou que, no $\mathrm{HGH}$ e LR, nos quatro tratamentos estudados, em torno de $80 \%$ do $\mathrm{N}$ mineral encontra-se como $\mathrm{N}$ nítrico (Fig. 2). Nos solos LV1 e, principalmente, no LV2 foi observado uma redução nos níveis de nitrato, constatando-se, inclusive, predominância do amônio no último solo. Nos solos HGP e PV, as percentagens de 
$\mathrm{N}$ mineral como nitrato foram maiores nos tratamentos com calcário, verificando-se também nesses solos um estímulo à nitrificação no tratamento com P. No Cambissolo, o teor de nitrato no tratamento Test contribuiu com quase $40 \%$ do $\mathrm{N}$ mineral, sendo notado, com a calagem e/ou aplicação de $\mathrm{P}$, forte estímulo à nitrificação. A predominância de nitrato no HGH, HGP, LR, ou em tratamentos do PV e C, causa preocupação, pela grande mobilidade desta forma de $\mathrm{N}$ nos solos. Com vistas a um aumento na eficiência de uso de $\mathrm{N}$ nítrico pelas culturas, seria aconselhável a adoção de práticas de manejo que buscassem maior sincronização de períodos de maior disponibilidade de nitrato no solo com a fase de maior demanda de $\mathrm{N}$ pelas plantas. No campo, a disponibilidade de nitrato no solo poderia ser mais bem controlada pelo estabelecimento correto da época de aplicação do corretivo e pela escolha adequada do calcário, tendo como base, principalmente, o grau de reatividade deste. Em nível mais amplo, a intensidade da nitrificação depende do tipo de solo, dos teores de matéria orgânica, $\mathrm{N}$ total, Ca trocável e Al tóxico, da disponibilidade de substrato (amônio)

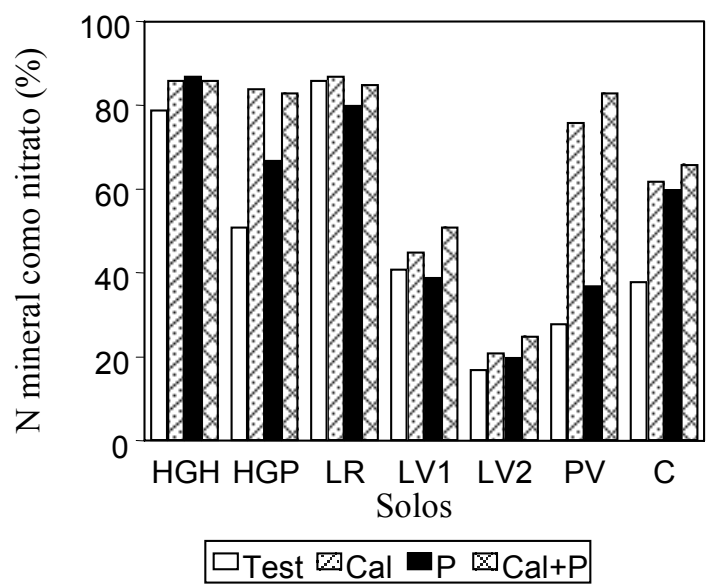

FIG. 2. Porcentagem de nitrogênio mineral na forma de nitrato sob influência dos tratamentos estudados. HGH: Glei Húmico; HGP: Glei Pouco Húmico; LR: Latossolo Roxo; LV1: Latossolo Vermelho-Amarelo (Itumirim); LV2: Latossolo Vermelho-Amarelo (Rio de Janeiro); PV: Podzólico Vermelho-Amarelo; C: Cambissolo. no solo e, por conseguinte, da atividade e população de nitrificadores (Haynes, 1986), o que, de certa forma, explica os padrões distintos de oxidação de amônio a nitrato observados nos sete solos estudados, ou em tratamentos destes.

As quantidades líquidas de $\mathrm{S}$ mineralizado foram influenciadas pelos tratamentos e tipos de solo, no decorrer dos 70 dias de incubação (Fig. 3). A prática da calagem nos solos HGP e LV2 resultou em maior intensidade na mineralização do $\mathrm{S}$. Os resultados obtidos estão de acordo com os apresentados por Nor \& Tabatabai (1977), Wainwright (1984) e Alvarez (1988) e evidenciam ser as bactérias do grupo Thiobacillus spp. (principais grupos de microrganismos envolvidos na oxidação do S) mais ativas em condições de $\mathrm{pH}$ próximas da neutralidade (Alexander, 1977). A resposta do processo de mineralização do $\mathrm{S}$ à aplicação de calcário esteve associada aos solos com maiores teores de $\mathrm{S}$ total, à exceção do $\mathrm{LR}$, onde os teores de $\mathrm{S}$ mineralizado foram muito baixos. No LR, verificou-se, inclusive, imobilização líquida de sulfato nos tratamentos Test e P. No PV, as quantidades líquidas de S mineralizado foram maiores do que as verificadas no LR, sendo verificada uma tendência de estímulo na oxidação do S orgânico nos tratamentos com calcário.

Os maiores teores de sulfato observados após a calagem do HGP e LV2 (Fig. 2) podem significar maior disponibilidade de $\mathrm{S}$ para as plantas, mas em condições que favoreçam maior movimentação de $\mathrm{S}$ no perfil, a maior disponibilidade de $\mathrm{S}\left(\mathrm{SO}_{4}{ }^{2-}\right)$ pode resultar em maior perda de $\mathrm{S}$ do sistema solo-planta, já que a calagem, pelo estímulo positivo que exerce sobre a densidade de cargas negativas nas argilas e óxidos de ferro e alumínio, diminui a adsorção de sulfato na camada de aração e aumenta a migração desse ânion para o subsolo (Alvarez, 1988).

Nos solos HGH, LV1 e C, o processo de mineralização do $\mathrm{S}$ não se mostrou influenciado pela aplicação de calcário e $\mathrm{P}$, sendo verificados, nesses solos, pequenas quantidades de $\mathrm{S}$ mineralizado, ou imobilização líquida de sulfato. Coincidentemente, esses são os solos com menores reservatórios de S total, sendo o LV1 e o C classificados entre os mais arenosos. Tais dados evidenciam a baixa capacidade dos solos HGH, LV1 e C em suprir S às plan- 
tas, permitindo a inferência de que o cultivo deles necessariamente dar-se-á com a adição de adubos contendo $\mathrm{S}$ e com a adoção de práticas agrícolas que visem à maior preservação do $\mathrm{S}$ no sistema solo-planta.
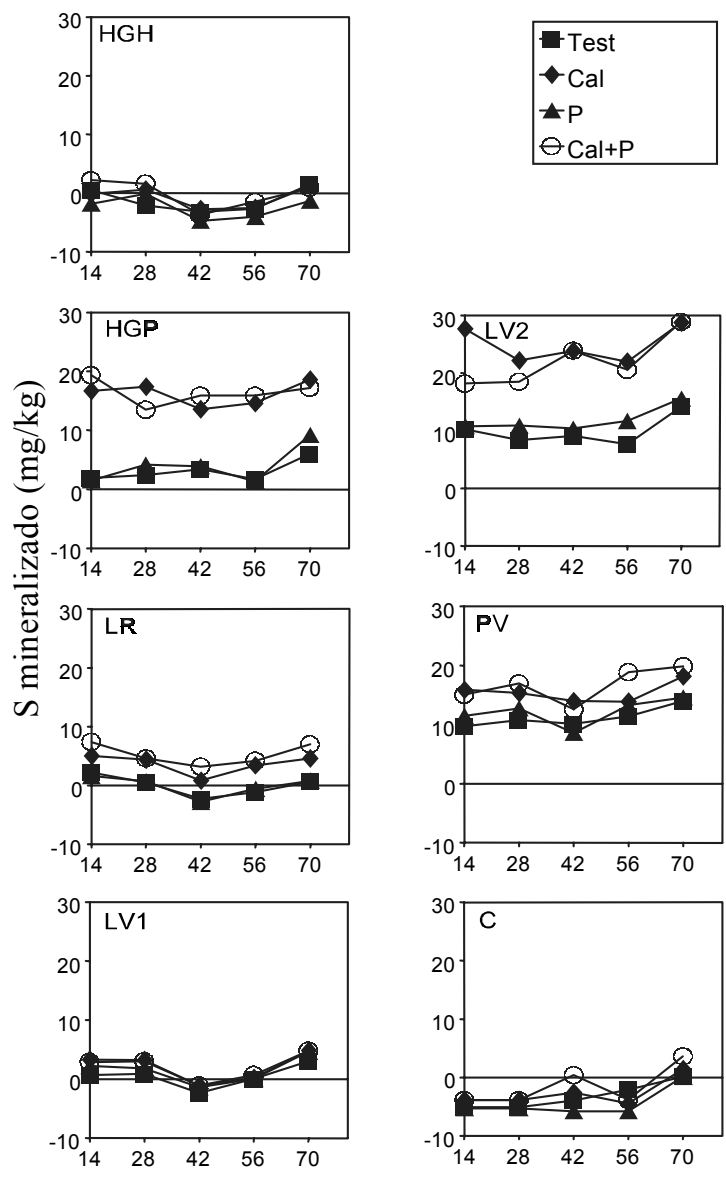

Período de incubação (dias)

FIG. 3. Mineralização líquida de enxofre, ao término dos cinco períodos de incubação, sob influência da calagem e da adição de fósforo. HGH: Glei Húmico; HGP: Glei Pouco Húmico; LR: Latossolo Roxo; LV1: Latossolo Vermelho-Amarelo (Itumirim); LV2: LatossoloVermelho-Amarelo (Rio de Janeiro); PV: Podzólico Vermelho-Amarelo; C: Cambissolo.
A maior disponibilidade de $\mathrm{P}$ nos sete materiais de solos estudados não resultou em maior conversão do S orgânico a sulfato; ao contrário, a associação da calagem com maiores teores disponíveis de P no LV2 acarretou maior imobilização de sulfato, pelo menos até os 42 dias de incubação.

Analisados de forma conjunta, os processos de mineralização de $\mathrm{N}$ e $\mathrm{S}$ apresentaram dinâmica diferenciada, ou seja: os teores de $\mathrm{N}$ mineralizado experimentaram maiores variações do que os de sulfato. Nesse sentido, apesar de se ter notado diminuição nas taxas de mineralização de $\mathrm{N}$ com o avanço do período de incubação, as quantidades líquidas de $\mathrm{N}$ mineralizado foram, na maioria dos solos, crescentes. Tal não foi o caso do $\mathrm{S}$, na medida em que, após 14 dias de incubação, as taxas de mineralização desse nutriente reduziram drasticamente, sendo os níveis de sulfato mais ou menos constantes com o avanço do período de incubação. Uma possível explicação para esse comportamento reside no fato de as formas de $\mathrm{S}$ orgânico presentes nos solos serem mineralizadas, independentemente do nível de demanda da biomassa microbiana por C (Sakadevan et al., 1993). McGill \& Cole (1981) sugerem também que a taxa e a extensão da mineralização de $\mathrm{S}$ é menos dependente da mineralização de $\mathrm{C}$ do que o processo de mineralização do $\mathrm{N}$, já que o $\mathrm{S}$ pode ser também liberado da matéria orgânica por processos bioquímicos, via hidrólise enzimática de ésteres de sulfato. Compartimentalizando o efeito da correção da acidez do solo, mais uma vez notam-se padrões de mineralização de $\mathrm{N}$ e $\mathrm{S}$ distintos, com a calagem promovendo um incremento nas quantidades de $\mathrm{S}$ mineralizado no HGP e LV2; tal não é o caso das quantidades de $\mathrm{N}$ mineralizado nesses dois solos.

\section{CONCLUSÕES}

1. A calagem promove uma aceleração na mineralização de N no HGH e LV2, e não concorre para um aumento de $\mathrm{N}$ mineral nos demais solos.

2. A correção da acidez do solo influencia a mineralização de S no HGP e LV2, e promove maior disponibilidade de sulfato nesses solos.

3. É muito baixa a capacidade dos solos $\mathrm{HGH}, \mathrm{LR}$, LV1 e $\mathrm{C}$ em suprir $\mathrm{S}$ e do LV1 e $\mathrm{C}$ em suprir $\mathrm{N}$ às plantas. 
4. A mineralização de $\mathrm{N}$ e $\mathrm{S}$ não é dependente dos níveis de $\mathrm{P}$ disponível nos sete solos estudados.

\section{REFERÊNCIAS}

ALEXANDER, M. Introduction to soil microbiology. 2.ed. New York: John Wiley, 1977. 467p.

ALVAREZ, V.H. Enxofre: critérios de diagnose para solo e planta, correção de deficiências e excessos. In: BORKERT, C.M.; LANTMANN, A.F. (Eds.). Enxofre e micronutrientes na agricultura brasileira. Londrina: Embrapa-CNPSo/IAPAR/ SBCS, 1988. p.31-59.

BARDSLEY, C.E.; LANCASTER, J.D. Determination of sulfur and soluble sulfates in soils. Soil Science Society of America. Proceedings, Madison, v.24, n.2, p.265-268, Feb. 1960.

BLACK, C.A. Soil-plant relationships. New York: John Wiley, 1968. 792p.

BREMNER, J.M.; MULVANEY, R.L. Nitrogen total. In: PAGE, A.L. (Ed.). Methods of soil analysis: chemical and microbiological properties. 2.ed. Madison: ASA/SSSA, 1982. p.595-622.

CAMARGO, O.A.; MONIZ, A.C.; JORGE, J.A.; VALADARES, J.M.A.S. Métodos de análise química, mineralógica e física de solos do Instituto Agronômico de Campinas. Campinas: Instituto Agronômico, 1986. 94p.

COELHO, A.M. Mineralização e nitrificação do nitrogênio em amostras do perfil de um Latossolo Vermelho-Escuro fase cerrado. Relatório Técnico Anual do Centro Nacional de Pesquisa de Milho e Sorgo, Sete Lagoas, v.5, p.216, 1992.

DANCER, W.S.; PETERSON, L.A.; CHESTERS, G. Ammonification and nitrification of $\mathrm{N}$ as influenced by soil $\mathrm{pH}$ and previous $\mathrm{N}$ treatments. Soil Science Society of America. Proceedings, Madison, v.37, n.1, p.67-69, 1973.

EMBRAPA. Serviço Nacional de Levantamento e Conservação do Solo. (Rio de Janeiro, RJ). Manual de métodos de análises de solo. Rio de Janeiro, 1979. $247 p$.

FALKINER, R.A.; KHANNA, P.K.; RAISON, R.J. Effect of superphosphate addition on $\mathrm{N}$ mineralization in some Australian forest soils. Soil Biology and Biochemistry, Oxford, v.31, n.2, p.285-296, Feb. 1993.

GOEDERT, W.J. Management of the cerrado soils of Brazil: a review. Journal of Soil Science, Oxford, v.34, n.3, p.405-428, Sept. 1983.

GUADALIX, M.E.; PARDO, M.T. Effects of liming on soil properties and $\mathrm{P}$ solubility in some Spanish Andosols. Geoderma, Amsterdam, v.63, p.53-61, 1994.

HAYNES, R.J. Nitrification. In: HAYNES, R.J. (Ed.). Mineral nitrogen in the plant-soil system. Madison: Academic, 1986. p.127-165.

KEENEY, D.R.; NELSON, D.W. Nitrogen-inorganic forms. In: PAGE, A.L. (Ed.). Methods of soil analysis: chemical and microbiological properties. 2.ed. Madison: ASA/SSSA, 1982. ch.33, p.643-698.

LOPES, A.S. Solos sob cerrado: características, propriedades e manejo. Piracicaba: Instituto da Potassa \& Fosfato/Instituto Internacional da Potassa, 1983. $162 \mathrm{p}$.

MARSCHNER, B.; WILCZYNSKI, A.W. The effect of liming on quantity and chemical composition of organic matter in pine forest in Berlin, Germany. Plant and Soil, The Hague, v.137, n.2, p.229-236, Nov. 1991.

McGILL, W.B.; COLE, C.V. Comparative aspects of cycling of organic $\mathrm{C}, \mathrm{N}, \mathrm{S}$ and $\mathrm{P}$ through soil organic matter. Geoderma, Amsterdam, v.26, p.267-286, 1981.

MENDONÇA, E.S.; ROWELL, D.L. Dinâmica do alumínio e de diferentes frações orgânicas de um latosssolo argiloso sob cerrado e soja. Revista Brasileira de Ciência do Solo, Campinas, v.18, n.3, p.295-303, maio/ago. 1994.

MIYAZAWA, M.; PAVAN, M.A.; CALEGARI, A. Efeito de material vegetal na acidez do solo. Revista Brasileira de Ciência do Solo, v.17, p.411-416, 1993.

MUNEVAR, F.; WOLLUM, A.G. Effects of the addition of phosphorus and inorganic nitrogen on carbon and nitrogen mineralization in Andepts from Colombia. Soil Science Society of America. Journal, Madison, v.41, n.3, p.540-545, May/July 1977. 
NASCIMENTO, J.A.L.; MORELLI, M. Enxofre em solos do Rio Grande do Sul. I. Formas nos solos. Revista Brasileira de Ciência do Solo, Campinas, v.4, n.3, p.131-135, set./dez. 1980.

NOR, Y.M.; TABATABAI, M.A. Oxidation of elemental sulfur in soils. Soil Science Society of America. Proceedings, Madison, v.41, n.4, p.736-741, July/Aug. 1977.

NYBORG, M.; HOYT, P.B. Effects of soil acidity and liming on mineralization of soil nitrogen. Canadian Journal of Soil Science, Ottawa, v.58, p.331-338, 1978.

RAIJ, B. van; QUAGGIO, J.A. Métodos de análise de solo para fins de fertilidade. Campinas: IAC, 1983. 31p. (IAC. Boletim técnico, 81).

SAKADEVAN, K.; HEDLEY, M.J.; MACKAY, A.D. Sulphur cycling in New Zealand hill country pastures. I. Laboratory sulphur, nitrogen and carbon mineralization studies. Journal of Soil Science, Oxford, v.44, n.1, p.73-83, Mar. 1993.
SCHNITZER, M.; KHAN, S.U. Soil organic matter. Amsterdam: Elsevier Scientific, 1972. 319p.

SILVA, C.A.; VALE, F.R.; GUILHERME, L.R.G. Efeito da calagem na mineralização do nitrogênio em solos de Minas Gerais. Revista Brasileira de Ciência do Solo, Campinas, v.18, n.3, p.471-476, set./dez. 1994.

SMITH, C.J.; GOH, K.M.; BOND, W.J.; FRENEY, J.R. Effets of organic and inorganic calcium compounds on soil-solution $\mathrm{pH}$ and aluminium concentration. European Journal of Soil Science, v.46, p.53-63, 1995.

STANFORD, G.; SMITH, S.J. Nitrogen mineralization potentials of soils. Soil Science Society of America. Proceedings, Madison, v.36, n.2, p.465472, 1972.

TABATABAI, M.A.; AL-KHAFAJI, A.A. Comparison of nitrogen and sulfur mineralization in soils. Soil Science Society of America. Journal, Madison, v.44, n.3, p.1000-1006, 1980.

WAINWRIGHT, M. Sulfur oxidation in soils. Advances in Agronomy, San Diego, v.37, p.349-396, 1984. 